\title{
Effects of Ursodeoxycholic Acid on Synthesis of Cholesterol and Bile Acids in Healthy Subjects
}

\author{
Gerd H. Sauter $^{a}$ Kai Thiessen ${ }^{a}$ Klaus G. Parhofer ${ }^{a}$ Christoph Jüngst $^{b}$ \\ Sven Fischer ${ }^{a}$ Dieter Jüngst ${ }^{a}$ \\ aDepartment of Medicine II, Klinikum Grosshadern, Ludwig Maximilians University, Munich, and \\ bDepartment of Medicine I, Rheinische Friedrich Wilhelms University, Bonn, Germany
}

\section{Key Words}

Bile acid synthesis · Cholesterol metabolism · Serum lipids . Ursodeoxycholic acid

\begin{abstract}
Background/Aims: Ursodeoxycholic acid (UDCA) decreases biliary secretion of cholesterol and is therefore used for the dissolution of cholesterol gallstones. It remains unclear whether these changes in biliary cholesterol excretion are associated with changes in cholesterol synthesis and bile acid synthesis. We therefore studied the activities of rate-limiting enzymes of cholesterol synthesis and bile acid synthesis, 3-hydroxy-3-methylglutaryl-coenzyme $A$ reductase and cholesterol $7 \alpha$-hydroxylase, respectively, in normal subjects during UDCA feeding. Methods: UDCA was given to 8 healthy volunteers ( 5 men, 3 women; age $24-44$ years) in a single dose of $10-15 \mathrm{mg} / \mathrm{kg}$ body weight for 40 days. Before and during (days 3, 5, 10, 20, 30 and 40) UDCA treatment, urinary excretion of mevalonic acid and serum concentrations of $7 \alpha$-hydroxy-4-cholesten-3-one $(7 \alpha$-HCO) were determined as markers of cholesterol and bile acid synthesis, respectively. The Wilcoxon signed rank test and Spearman's rank correlation coefficient were used for statistical analysis. Results: Cholesterol synthesis and serum lipid concentrations remained unchanged during UDCA treatment for 40 days. However, synthesis of bile acids
\end{abstract}

\section{KARGER}

Fax +41613061234 E-Mail karger@karger.ch www. karger.com
(C) 2004 S. Karger AG, Basel

0012-2823/04/0702-0079\$21.00/0

Accessible online at:

www. karger.com/dig increased during long-term treatment with UDCA as reflected by an increase in $7 \alpha-\mathrm{HCO}$ serum concentrations from $39.7 \pm 21.3 \mathrm{ng} / \mathrm{ml}$ (median $32.8 \mathrm{ng} / \mathrm{ml}$ ) before treatment to $64.0 \pm 30.4 \mathrm{ng} / \mathrm{ml}$ (median $77.5 \mathrm{ng} / \mathrm{ml}$ ) at days 30-40 of UDCA treatment $(p<0.05)$. Conclusions: UDCA treatment does not affect cholesterol synthesis in the liver, but does increase bile acid synthesis after prolonged treatment. This may represent a compensatory change following decreased absorption of endogenous bile acids as observed with UDCA therapy.

Copyright $(\subset) 2004$ S. Karger AG, Basel

\section{Introduction}

In patients with cholesterol gallstones, treatment with ursodeoxycholic acid (UDCA) decreases biliary secretion of cholesterol and cholesterol saturation of gallbladder bile [1, 2]. Moreover, impaired intestinal absorption of cholesterol has been found by some [3-5], but not all authors [6, 7] during UDCA treatment. It remains unclear, however, whether UDCA therapy also affects cholesterol metabolism. Both stimulatory [8] and inhibitory effects of UDCA on 3-hydroxy-3-methylglutaryl-coenzyme A (HMG-CoA) reductase [9, 10], the rate-limiting enzyme of cholesterol synthesis, have been reported in patients with gallstones. The enzymatic activity as well as mRNA levels of cholesterol $7 \alpha$-hydroxylase, the rate-lim- 
iting enzyme of the catabolic conversion of cholesterol into bile acids, were found to be unchanged in human liver tissue after short-term treatment with UDCA [11, 12]. However, increased bile acid synthesis rates were observed in one study after ingestion of UDCA for 6 weeks [1]. Studies in rats have shown that mRNA levels of HMG-CoA reductase and cholesterol $7 \alpha$-hydroxylase do not change significantly during UDCA feeding [13], while enzymatic activities of HMG-CoA reductase in liver microsomes may increase [14]. In primary human hepatocytes incubated with UDCA, mRNA levels of cholesterol 7a-hydroxylase remained unchanged [15]. Hence, it remains unclear whether UDCA, either directly by its pharmacological action or secondary to effects on biliary excretion or intestinal absorption of cholesterol, affects the hepatic metabolism of cholesterol.

We therefore studied the activities of two key enzymes of cholesterol and bile acid synthesis during ingestion of UDCA in normal subjects. Urinary excretion of mevalonic acid (MVA), the enzymatic product of HMG-CoA reductase, has been shown to reliably reflect HMG-CoA reductase activity in the liver and was used as a semiquantitative marker of cholesterol synthesis [16]. The activity of cholesterol $7 \alpha$-hydroxylase was assessed by determination of serum concentrations of $7 \alpha$-hydroxy-4-cholesten3 -one $(7 \alpha-\mathrm{HCO})$, an intermediate of the major metabolic pathway of bile acid synthesis $[17,18]$. In addition, we evaluated the effects of UDCA on serum lipid and apolipoprotein (Apo) concentrations.

\section{Subjects and Methods}

\section{Subjects and Study Protocol}

UDCA was given to 8 healthy subjects ( 5 men, 3 women; age 24-44 years) for 40 days as a single dose of $10-15 \mathrm{mg} / \mathrm{kg}$ body weight in the evening. The body weight of the subjects was between 54 and $95 \mathrm{~kg}$ (median $77 \mathrm{~kg}$ ), with a body mass index between 21.4 and $30.7 \mathrm{~kg} / \mathrm{m}^{2}$ (median $23.2 \mathrm{~kg} / \mathrm{m}^{2}$ ). Blood was drawn in the morning after a fasting period overnight, and urine was collected overnight for $12 \mathrm{~h}$ on days $3,5,10,20,30$ and 40 of UDCA treatment. Mean values from two time points (before treatment, days 3 and 5, days 10 and 20, days 30 and 40) were used for further calculations. At all of these time points, $20 \mathrm{ml}$ of serum was preserved for determinations of serum lipoprotein profiles, Apo B and Apo A1.

\section{Analytical Methods}

Urinary MVA was assayed as a marker of whole-body cholesterol synthesis by combined gas chromatography-mass spectrometry using the isotope dilution method described by Lindenthal et al. [16] and Lindenthal and von Bergmann [19]. Briefly, $100 \mathrm{ng}$ of ${ }^{2} \mathrm{H}_{7}$-MVA (MSD Isotopes, Montreal, Canada) was added as internal standard to $400 \mu \mathrm{l}$ of urine. After extraction, purification and derivatization with methyl-tertiary-butyldimethylsilyl-trifluoroacetamide, the concentration of MVA was analyzed by gas chromatography-mass spectrometry on a Finnigan 4000 quadrupole mass spectrometer, SIM mode. The ion $\mathrm{m} / \mathrm{z} 433$ (base peak) was scanned in the selected ion monitoring mode for endogenous MVA and the ion $\mathrm{m} / \mathrm{z} 440$ for ${ }^{2} \mathrm{H}_{7-}$ MVA. Creatinine in urine was measured by a standard laboratory procedure.

$7 \alpha-\mathrm{HCO}$ in serum as a marker of bile acid synthesis was analyzed by high-performance liquid chromatography (HPLC) according to the method of Axelson et al. [20]. Briefly, $7 \alpha-\mathrm{HCO}$ was extracted in jacketed glass columns connected to a water bath on octadecylsilanebonded silica at $64^{\circ} \mathrm{C}$. After a wash with water and $65 \%$ aqueous methanol, $7 \alpha-\mathrm{HCO}$ was eluted with hexane-chloroform $(80: 20$, vol/ vol). Analysis was performed by HPLC on a column $(250 \times 4.5 \mathrm{~mm})$ of LiChrospher 100 RP-18 (Merck, Darmstadt, Germany) connected to a UV detector (SPD-10A, Shimadzu, Kyoto, Japan) at a wavelength of $254 \mathrm{~nm}$. Acetonitrile-water $(98: 2, \mathrm{vol} / \mathrm{vol})$ was used as the mobile phase at a flow rate of $1 \mathrm{ml} / \mathrm{min}$. Serum concentrations of $7 \alpha-\mathrm{HCO}$ were calculated from known amounts of $7 \beta-\mathrm{HCO}$ as internal standard.

Very-low-density lipoproteins (VLDL) were separated by ultracentrifugation $\left(45,000 \mathrm{rpm}, 20 \mathrm{~h}, 4^{\circ} \mathrm{C}\right.$, Beckman rotor Ti 50 , density $1.006 \mathrm{~g} / \mathrm{ml}$ ). High-density lipoprotein (HDL) cholesterol was determined in the infranatant after heparin-manganese precipitation of Apo B-containing lipoproteins. Low-density lipoprotein (LDL) cholesterol was calculated by subtracting HDL cholesterol from total infranatant cholesterol. Triglyceride and cholesterol levels in serum and lipoprotein fractions were measured enzymatically by an autoanalyzer (EPOS, Eppendorf, Hamburg, Germany) using reagents from Boehringer (Mannheim, Germany). Apo B and Apo A1 concentrations were determined by nephelometry (Behring, Marburg, Germany) using antibodies against human Apo B and Apo A1 (anti-Apo B, anti-Apo A1 from rabbit, Dade Behring, Marburg, Germany).

Data are given as mean \pm SD or median. The Wilcoxon signed ranks test for paired samples and Spearman's rank correlation coefficient were used for statistical analysis. Data analysis was performed with the SPSS for Windows program 10.0 (SPSS Inc., Chicago, Ill., USA). $p$ values of less than 0.05 were considered statistically significant.

\section{Results}

Concentrations of urinary MVA were not affected by UDCA treatment (fig. 1). In contrast, serum concentrations of $7 \alpha-\mathrm{HCO}$ increased from $39.7 \pm 21.3 \mathrm{ng} / \mathrm{ml}$ (median $32.8 \mathrm{ng} / \mathrm{ml}$ ) before treatment to $64.0 \pm 30.4 \mathrm{ng} / \mathrm{ml}$ (median $77.5 \mathrm{ng} / \mathrm{ml}$ ) at days 30-40 of UDCA treatment $(p<0.05)$ (fig. 2). Serum lipoprotein concentrations (cholesterol, triglycerides, LDL cholesterol, HDL cholesterol, VLDL cholesterol) remained unchanged with the exception of VLDL triglycerides, which transiently decreased from 85 to $64 \mathrm{mg} / \mathrm{dl}$ at days 10-20 of UDCA treatment $(\mathrm{p}<0.05)$ (table 1). Before treatment, serum concentrations of VLDL triglycerides were positively correlated with serum concentrations of MVA $(n=8 ; r=0.79, p=$ 


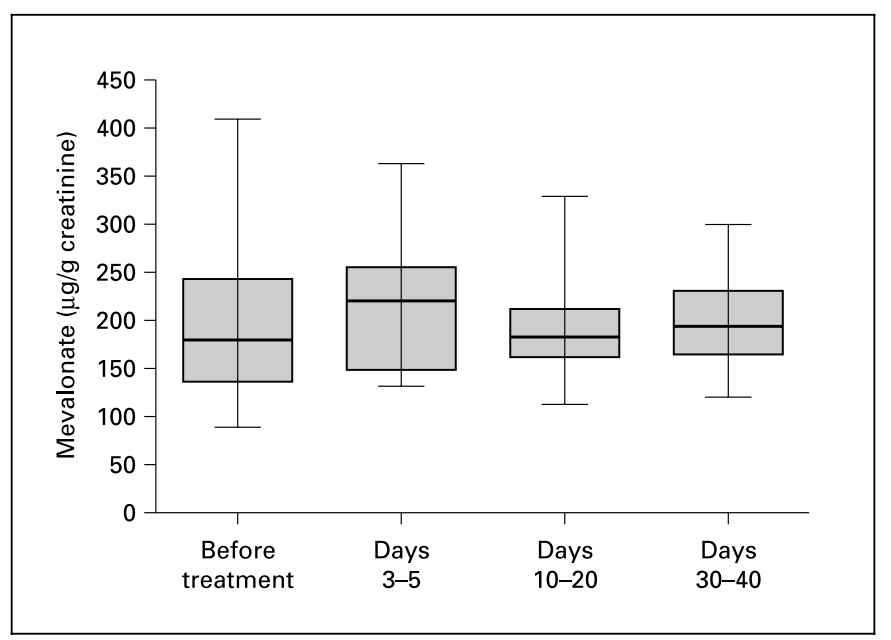

Fig. 1. Urinary MVA excretion as a marker of whole-body biosynthesis of cholesterol before and during treatment with UDCA (box and whiskers plots). Cholesterol synthesis remained unchanged during UDCA feeding.

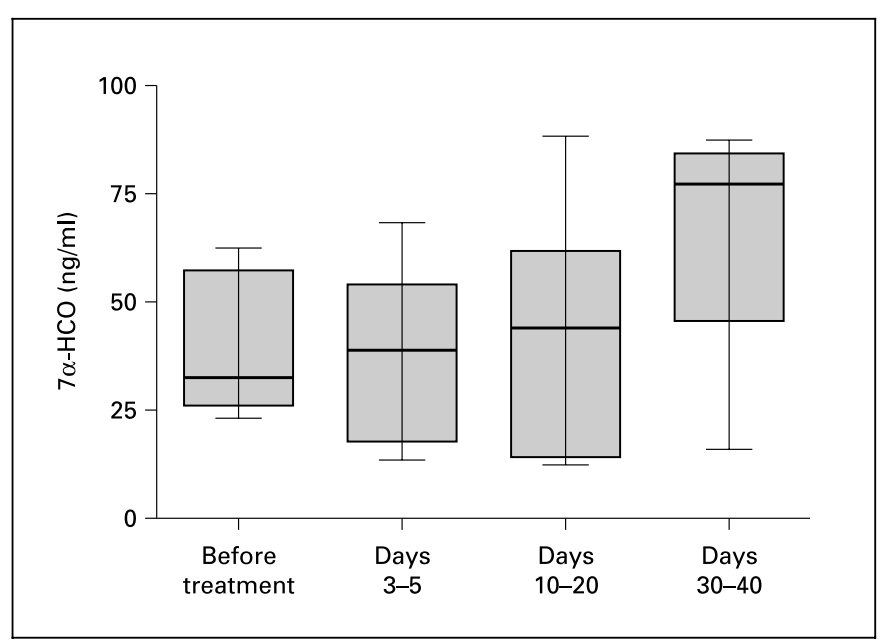

Fig. 2. Serum levels of $7 \alpha-\mathrm{HCO}$ as a marker of bile acid synthesis before and during UDCA feeding (box and whiskers plots). Serum levels of $7 \alpha-\mathrm{HCO}$ remained constant within the first 3 weeks of UDCA treatment but increased at days 30-40.
Table 1. Serum concentrations of cholesterol, triglycerides, LDL cholesterol, HDL cholesterol, VLDL cholesterol, VLDL triglycerides, Apo A1 and Apo B of 8 volunteers who ingested UDCA for 40 days

\begin{tabular}{lcccc}
\hline & $\begin{array}{c}\text { Before } \\
\text { treatment }\end{array}$ & Days 3-5 & Days 10-20 & Days 30-40 \\
& $172 \pm 31$ & $170 \pm 26$ & $173 \pm 21$ & $171 \pm 22$ \\
Cholesterol, mg/dl & $92 \pm 50$ & $86 \pm 33$ & $91 \pm 28$ & $98 \pm 22$ \\
Triglycerides, mg/dl & $54 \pm 13$ & $53 \pm 13$ & $58 \pm 13$ & $55 \pm 11$ \\
HDL cholesterol, mg/dl & $104 \pm 26$ & $101 \pm 24$ & $99 \pm 21$ & $100 \pm 21$ \\
LDL cholesterol, mg/dl & $13 \pm 5$ & $15 \pm 5$ & $15 \pm 6$ & $17 \pm 5$ \\
VLDL cholesterol, mg/dl & $85 \pm 43$ & $66 \pm 34$ & $64 \pm 28^{*}$ & $75 \pm 19$ \\
VLDL triglycerides, mg/dl & $157 \pm 25$ & $155 \pm 23$ & $164 \pm 32$ & $161 \pm 27$ \\
Apo A1, mg/dl & $91 \pm 18$ & $85 \pm 19$ & $86 \pm 19$ & $87 \pm 17$ \\
Apo B, mg/dl & & & & \\
\hline
\end{tabular}

Data are given as mean $\pm \mathrm{SD} .{ }^{*} \mathrm{p}<0.05$ compared to pretreatment values.
0.021). Correlations between MVA and $7 \alpha-\mathrm{HCO}$ in serum as well as correlations between each of these two parameters and body weight, body mass index and age did not reach statistical significance.

\section{Discussion}

In the present study, bile acid synthesis was found to increase in normal subjects after ingestion of UDCA for more than 4 weeks. The activity of HMG-CoA reductase, the rate-limiting enzyme of cholesterol synthesis, remained unchanged during UDCA treatment.
Our interpretations are based on the finding that treatment with UDCA for more than 4 weeks increased serum concentrations of $7 \alpha-\mathrm{HCO}$ in healthy subjects. Serum concentrations of $7 \alpha-\mathrm{HCO}$ have been shown to correlate closely with hepatic cholesterol $7 \alpha$-hydroxylase activity in human liver [17] as well as with total synthesis of primary bile acids as measured by the isotope dilution technique [18]. Hence, the present data provide evidence that UDCA enhances the conversion of cholesterol into bile acids after prolonged treatment. Using the synthetic bile acid analogue ${ }^{75}$ selenium homocholic acid taurine, Eusufzai et al. [21] found a dose-dependent reduction of intestinal bile acid reabsorption during administration of UDCA for 3 weeks. Moreover, Stiehl et al. [22] reported 
impaired absorption of endogenous bile acids in patients with ileostomies who had received a single dose of UDCA. These findings are in agreement with kinetic studies, which have shown increased fractional turnover rates of cholic acid and chenodeoxycholic acid during UDCA treatment [1, 2, 4, 23, 24]. These kinetic studies have also shown that pool sizes of endogenous bile acids decrease with UDCA treatment. However, there are conflicting data regarding changes in bile acid synthesis, which would be expected to increase in order to compensate for the increased loss of bile acids. While bile acid synthesis was reported to increase by $40-80 \%$ during UDCA treatment for 5-6 weeks in one study [1], unchanged cholesterol $7 \alpha$-hydroxylase activity was found under short-term conditions [8, 12, 17], and even decreased synthesis rates have been reported [23]. Hardison and Grundy [4] used a balance technique in which they measured both the daily fecal output of bile acids and the incorporation of injected $\left[{ }^{14} \mathrm{C}\right]$ cholesterol into bile acids. They found that mean total bile acid synthesis increased from 650 to $800 \mathrm{mg} /$ day when UDCA ( $15 \mathrm{mg} / \mathrm{kg}$ day) was administered for 1 month.

In the present study, bile acid synthesis remained unchanged within the first weeks of the study, but increased after more than 4 weeks. This finding supports the hypothesis that the effects of UDCA on cholesterol and bile acid metabolism may change over time [8]. The lack of shortterm effects indicates that UDCA either has no direct effect on bile acid synthesis or moderately inhibits bile acid synthesis, thereby neutralizing the stimulatory effect of bile acid malabsorption in the first weeks of UDCA treatment. This hypothesis is in agreement with the fact that UDCA binds only weakly to the farnesoid $\mathrm{X}$ receptor, which has been shown to inhibit cholesterol $7 \alpha$-hydroxylase gene expression after binding of endogenous bile acids $[25,26]$. Since UDCA treatment results in a continuous loss of endogenous bile acids, the observed increase in cholesterol $7 \alpha$-hydroxylase activity is probably due to an increasing shortage of endogenous bile acids over time.

Whole-body cholesterol synthesis as measured by urinary mevalonate excretion remained unchanged during the 40-day regimen of UDCA treatment in our study. This finding is in agreement with data of Angelin et al. [27], who observed unchanged hepatic HMG-CoA reductase activity in patients with gallstones after 6 weeks of treatment with UDCA. However, Maton et al. [9] found that hepatic HMG-CoA reductase activity was reduced to $35 \%$ after 3-5 months of UDCA treatment, and Salen et al. [10] reported a threefold decrease in 2 patients after 1 year of UDCA treatment. Even though data on changes in hepatic HMG-CoA reductase activity after longer treatment periods are conflicting, decreased biliary excretion of cholesterol obviously does not affect cholesterol synthesis under short-term conditions. These findings correspond to recent observations of Hillebrant et al. [28], who studied the effects of combined treatment with pravastatin and UDCA on hepatic cholesterol metabolism. Pravastatin did not further reduce the cholesterol saturation of bile in patients with gallstones treated with UDCA, although hepatic cholesterol synthesis was inhibited. Our results support the concept that de novo synthesis of cholesterol is not particularly important for biliary cholesterol secretion in humans.

The effects of UDCA on serum lipoprotein profiles have mainly been studied in patients with gallstone disease. Erlinger et al. [29] treated 197 patients at daily doses of $2.1-16.2 \mathrm{mg} / \mathrm{kg}$ for up to 1 year and observed no significant influence of UDCA treatment on serum cholesterol and triglycerides. Fromm et al. [30] found no change in serum cholesterol and triglyceride in serum in patients with gallstones who were fed UDCA for 1 year at doses of $400-800 \mathrm{mg} /$ day. In the present study on normal subjects, we were unable to detect changes in cholesterol and triglyceride in serum, including LDL cholesterol, HDL cholesterol and VLDL cholesterol. However, there was a transient decrease in VLDL triglycerides at days 10-20. This finding is in agreement with studies demonstrating an inhibitory effect of bile acids, including UDCA, on VLDL lipoprotein secretion in rat hepatocytes in primary culture [31]. Moreover, serum concentrations of VLDL triglycerides were positively correlated with serum concentrations of MVA before, but not during UDCA treatment. A similar correlation between cholesterol synthesis and secretion of VLDL Apo B was described by Watts et al. [32] in normolipidemic subjects, indicating that in vivo cholesterol synthesis is a determinant of hepatic secretion of Apo B.

In conclusion, the present study shows that ingestion of UDCA moderately increases bile acid synthesis after treatment periods of 4-6 weeks. Moreover, from the transient decrease in VLDL triglycerides during UDCA feeding, we cannot exclude the possibility that UDCA affects VLDL secretion.

\section{Acknowledgements}

The authors are grateful to Gudrun Schwertfeger for technical assistance, and to Viera Stefanek and Birgit Eberlein for secretarial assistance. This study was supported by a grant of the Else KrönerFresenius Foundation, Homburg Saar, Germany, and by the Falk Foundation, Freiburg, Germany. 


\section{References}

1 Nilsell K, Angelin B, Leijd B, Einarsson K: Comparative effects of ursodeoxycholic acid and chenodeoxycholic acid on bile acid kinetics and biliary lipid secretion in humans. Evidence for different modes of action on bile acid synthesis. Gastroenterology 1983;85:1248-1256.

2 von Bergmann K, Epple-Gutsfeld M, Leiss O: Differences in the effects of chenodeoxycholic and ursodeoxycholic acid on biliary lipid secretion and bile acid synthesis in patients with gallstones. Gastroenterology 1984;87:136143.

3 Ponz-de-Leon M, Carulli N, Loria P, Iori R, Zironi F: Cholesterol absorption during bile acid feeding. Effect of ursodeoxycholic acid (UDCA) administration. Gastroenterology 1980;78:214-219

4 Hardison WG, Grundy SM: Effect of ursodeoxycholate and its taurine conjugate on bile acid synthesis and cholesterol absorption. Gastroenterology 1984;87:130-135.

5 Leiss O, von Bergmann K, Streicher U, Strotkoetter H: Effect of three different dihydroxy bile acids on intestinal cholesterol absorption in normal volunteers. Gastroenterology 1984; 87:144-149.

6 LaRusso NF, Thistle JL: Effect of litholytic bile acids on cholesterol absorption in gallstone patients. Gastroenterology 1983;84:265-271.

7 Woollett LA, Buckley DD, Yao L, et al: Effect of ursodeoxycholic acid on cholesterol absorption and metabolism in humans. J Lipid Res 2003;44:935-942.

8 Carulli N, Ponz-de-Leon M, Zironi F, et al: Hepatic cholesterol and bile acid metabolism in subjects with gallstones: Comparative effects of short term feeding of chenodeoxycholic and ursodeoxycholic acid. J Lipid Res 1980;21:3543.

9 Maton PN, Ellis HJ, Higgins MJ, Dowling RH: Hepatic HMG-CoA reductase in human cholelithiasis: Effects of chenodeoxycholic and ursodeoxycholic acids. Eur J Clin Invest 1980;10: 325-332.

10 Salen G, Colalillo A, Verga D, Bagan E, Tint GS, Shefer S: Effect of high and low doses of ursodeoxycholic acid on gallstone dissolution in humans. Gastroenterology 1980;78:14121418 .
11 Bertolotti M, Abate N, Loria P, et al: Regulation of bile acid synthesis in humans: Effect of treatment with bile acids, cholestyramine or simvastatin on cholesterol $7 \alpha$-hydroxylation rates in vivo. Hepatology 1991;14:831-837.

12 Rudling M, Angelin B, Ståhle L, Reihnér E, Sahlin S, Olivecrona H, Björkhem I, Einarsson C: Regulation of hepatic low-density lipoprotein receptor, 3-hydroxy-3-methylglutaryl coenzyme A reductase, and cholesterol $7 \alpha$ hydroxylase mRNAs in human liver. J Clin Endocrinol Metab 2002;87:4307-4313.

13 Shefer S, Kren BT, Salen G, et al: Regulation of bile acid synthesis by deoxycholic acid in the rat: Different effects on cholesterol $7 \alpha$-hydroxylase and sterol 27-hydroxylase. Hepatology 1995;22:1215-1221.

14 Honda A, Salen G, Nguyen LB, et al: Regulation of early cholesterol biosynthesis in rat liver: Effects of sterols, bile acids, lovastatin, and BM 15.766 on 3-hydroxy-3-methylglutaryl coenzyme A synthase and acetoacetyl coenzyme A thiolase activities. Hepatology 1998; 27:154-159.

15 Ellis E, Axelson M, Abrahamsson A, et al: Feedback regulation of bile acid synthesis in primary human hepatocytes: Evidence that CDCA is the strongest inhibitor. Hepatology 2003;38:930-938.

16 Lindenthal B, Simatupang A, Dotti MT, Federico A, Lütjohann D, von Bergmann K: Urinary excretion of mevalonic acid as an indicator of cholesterol synthesis. J Lipid Res 1996; 37:2193-2201.

17 Axelson M, Björkhem I, Reihnér E, Einarsson $\mathrm{K}$ : The plasma level of $7 \alpha$-hydroxy-4-cholesten3 -one reflects the activity of hepatic cholesterol $7 \alpha$-hydroxylase in man. FEBS Lett 1991;284: 216-218.

18 Sauter G, Berr F, Beuers U, Fischer S, Paumgartner G: Serum concentrations of 7 $7 \alpha$-hydroxy-4-cholesten-3-one reflect bile acid synthesis in man. Hepatology 1996;24:123-126.

19 Lindenthal B, von Bergmann K: Determination of urinary mevalonic acid using isotope dilution technique. Biol Mass Spectrom 1994; 23:445-450.

20 Axelson M, Aly A, Sjövall J: Levels of $7 \alpha-$ hydroxy-4-cholesten-3-one in plasma reflect rates of bile acid synthesis. FEBS Lett 1988; 239:324-328.

21 Eusufzai S, Ericsson S, Cederlund T, Einarsson K, Angelin B: Effect of ursodeoxycholic acid treatment on ileal absorption of bile acids in man as determined by the SeHCAT test. Gut 1991;32:1044-1048.
22 Stiehl A, Raedsch R, Rudolph G: Acute effects of ursodeoxycholic and chenodeoxycholic acid on the small intestinal absorption of bile acids. Gastroenterology 1990;98:424-428.

23 Roda E, Roda A, Sama C, et al: Effect of ursodeoxycholic acid administration on biliary lipid composition and bile acid kinetics in cholesterol gallstone patients. Dig Dis Sci 1979;24: 123-128.

24 Tint GS, Salen G, Shefer S: Effect of ursodeoxycholic acid and chenodeoxycholic acid on cholesterol and bile acid metabolism. Gastroenterology 1986;91:1007-1018.

25 Makishima M, Okamoto AY, Repa JJ, et al: Identification of a nuclear receptor for bile acids. Science 1999;284:1362-1365.

26 Parks DJ, Blanchard SG, Bledsoe RK, et al: Bile acids: Natural ligands for an orphan nuclear receptor. Science 1999;284:1365-1368.

27 Angelin B, Ewerth S, Einarsson K: Ursodeoxycholic acid treatment in cholesterol gallstone disease: Effects on hepatic 3-hydroxy-3-methylglutaryl coenzyme A reductase activity, biliary lipid composition, and plasma lipid levels. J Lipid Res 1983;24:461-468.

28 Hillebrant CG, Nyberg B, Gustafsson U, et al: Effects of combined treatment with pravastatin and ursodeoxycholic acid on hepatic cholesterol metabolism. Eur J Clin Invest 2002;32:528534.

29 Erlinger S, Le Go A, Husson JM, Fevery J: Franco-Belgian cooperative study of ursodeoxycholic acid in the medical dissolution of gallstones: A double-blind, randomized, doseresponse study, and comparison with chenodeoxycholic acid. Hepatology 1984;4:308-314.

30 Fromm H, Roat JW, Gonzalez V, Sarva RP Farivar S: Comparative efficacy and side effects of ursodeoxycholic and chenodeoxycholic acids in dissolving gallstones. A double-blind controlled study. Gastroenterology 1983;85: 1257-1264

31 Lin Y, Havinga R, Verkade HJ, et al: Bile acids suppress the secretion of very-low-density lipoprotein by human hepatocytes in primary culture. Hepatology 1996;23:218-228.

32 Watts GF, Naoumova R, Cummings MH, et al: Direct correlation between cholesterol synthesis and hepatic secretion of apolipoprotein B100 in normolipidemic subjects. Metabolism 1995;44:1052-1057. 